\title{
Capillary and van der Waals forces between uncharged colloidal particles linked by a liquid bridge
}

\section{Journal Article}

Author(s):

Megias-Alguacil, David; Gauckler, Ludwig J.

Publication date:

2010

Permanent link:

https://doi.org/10.3929/ethz-b-000017330

Rights / license:

In Copyright - Non-Commercial Use Permitted

Originally published in:

Colloid \& polymer science 288(2), https://doi.org/10.1007/s00396-009-2106-0 


\title{
Capillary and van der Waals forces between uncharged colloidal particles linked by a liquid bridge
}

\author{
David Megias-Alguacil $\cdot$ Ludwig J. Gauckler
}

Received: 3 July 2009 /Revised: 7 August 2009 /Accepted: 27 August 2009 /Published online: 16 September 2009

(C) Springer-Verlag 2009

\begin{abstract}
This work presents a theoretical study of the forces established between colloidal particles connected by means of a concave liquid bridge, where the solid particles are partially wetted by a certain amount of liquid also possessing a dry portion of their surfaces. In our analysis, we adopt a two-particle model assuming that the solids are spherical and with the same sizes and properties and that the liquid meniscus features an arc-of-circumference contour. The forces considered are the typical capillary ones, namely, wetting and Laplace forces, as well as the van der Waals force, assuming the particles uncharged. We analyze different parameters which govern the liquid bridge: interparticle separation, wetting angle, and liquid volume, which later determine the value of the forces. Due to the dual characteristic of the particles' surfaces, wet and dry, the forces are to be determined numerically in each case. The results indicate that the capillary forces are dominant in most of the situations meanwhile the van der Waals force is noticeable at very short distances between the particles.
\end{abstract}

Keywords Liquid bridge · Meniscus · Capillary forces . van der Waals

\section{Introduction}

The insertion of liquid film in between fine particles modifies the film geometry, which further forms a liquid bridge with a curved meniscus shape. The contact between

D. Megias-Alguacil $(\triangle) \cdot$ L. J. Gauckler

Non-Metallic Inorganic Materials, Department of Materials, ETH-Zürich,

8093 Zürich, Switzerland

e-mail: david.megias@mat.ethz.ch the three phases, solid, liquid, and gas, induces the onset of forces between these particles, which will depend on physical-chemical aspects like the wettability of the particles, the geometry of the meniscus as well as the particles size, and separation between them.

Capillary forces between pairs of particles due to a liquid bridge have been investigated by different authors, i.e., [1-13], since this is the scenario found in many practical situations like in wet granular media [14], particle stabilized foams [15], etc. and processes like liquid phase sintering [6]. Nevertheless, in these studies, typical colloidal forces are not usually considered even though the size of the particles may fall into the colloidal domain. This approach can lead in an insufficient description of the liquid bridge system, which could mask some results about the mechanics of the system. The aim of the present work is to contribute to this topic by studying the forces established between two colloidal particles in contact with a liquid bridge taking into account not only the capillary forces, but also the van der Waals force, as representative for the colloidal forces. In the present work, the particles are considered to be uncharged in order to avoid the introduction of the electrostatic interaction, as well as other forces of colloidal nature, which would add more complexity to such a study. Nevertheless, recall that there are situations like flotation, where the particles are confined at the airwater interface, in which the electrostatic interaction, if present, is very important or even the dominant force due to its strength and long range.

\section{System description}

Let us consider a linear string of solid monosized spherical colloidal particles, all spherical and with the same sizes and 
properties. The surface of the particles is assumed to be perfectly smooth and chemically homogeneous, thus the wetting angle, $\theta$, between the solid and liquid interfaces will be constant against any relative displacement of the particles, and any wetting hysteresis will not occur [16]. The particles are partially wetted by the liquid, which forms a concave liquid bridge between the spheres in the so-called pendular state (where the liquid phase is discontinuous). Considering that the particles' size is in the colloidal range, the effect of gravity is negligible, and no other buoyancy force will be considered. In such a case, the liquid bridge has a constant pressure [17], and the meniscus posses the shape of a surface of revolution [18, 19] with the same mean curvature everywhere [9]. The meniscus shape is defined as an arc of one of the Delaunay's surfaces [20], which are generated by the rotation around the basis of the Delaunay's roulettes [17]. Also, other axisymmetric profiles of uniform mean curvature, like the nodoid, catenoid, or unduloid, have been considered [5,9]. In the present work, for sake of simplicity, we will assume a meniscus profile described by an arc of circumference, as sketched in Fig. 1, under the assumption that the error brought about by this approximation is small in most cases $[6,9,18,21]$.

Considering, for sake of simplicity, just the upper right quadrant, the liquid and solid profiles are described, respectively, by:

$y_{L}(x)=(\rho+L)-\sqrt{\rho^{2}-x^{2}}$

and:

$y_{S}(x)=\sqrt{R^{2}-\left(x-\frac{H}{2}-R\right)^{2}}$

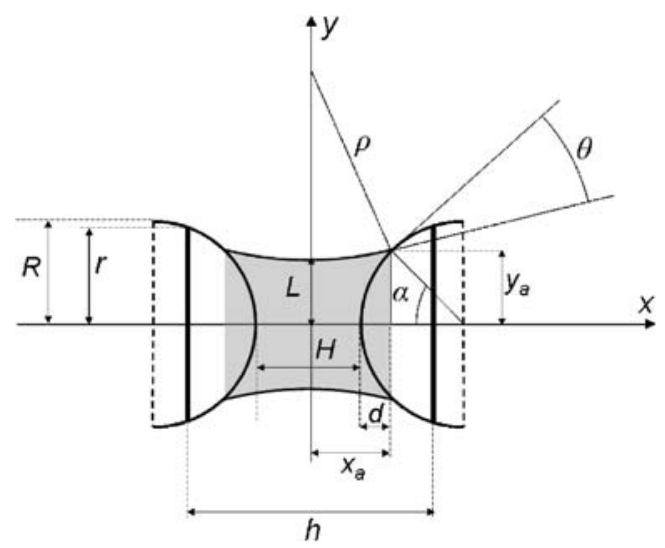

Fig. 1 Sketch of the liquid bridge geometry. $R$ is the solid particle's radius, $x_{a}$ and $y_{a}$ are the coordinates of the contact point between the solid and liquid profile, $\alpha$ is the half-filling angle, $\theta$ is the wetting angle, $\rho$ and $L$ are the principal radius of the liquid meniscus, $H$ is the surfaceto-surface distance between the solid particles, $d$ is the wetted portion of each hemisphere, $h$ is the distance between elements of integration used in the calculation of the van der Waals force, and $r$ their radius
The principal radii, $\rho$ and $L$, are described in terms of both representative angles, $\alpha$ and $\theta$, and the coordinates of the contact point:

$\rho=\frac{x_{a}}{\cos (\alpha+\theta)}$

and

$L=y_{a}+\rho[1-\sin (\alpha+\theta)]$

Notice that the principal radius $\rho$ is related to the concave-convex character of the liquid meniscus, meanwhile the radius $L$ gives an indication of the meniscus thickness.

The liquid volume of the bridge, $V$, may be determined for a given distance between particles, $H$, by definite integration of both the solid and liquid profiles:

$V=2 \pi \int_{0}^{x_{a}}\left[y_{L}(x)\right]^{2} d x-2 \pi \int_{H / 2}^{x_{a}}\left[y_{S}(x)\right]^{2} d x$

This volume must be calculated numerically for a certain separation $H$ and wetting angle $\theta$. Once this value is obtained, $\rho$ and $L$ are also determined. On what follows, we will refer to the liquid volume of the bridge throughout the relative volume, $V_{r e l}$, of the liquid with respect to the volume of the sphere, then, $V_{\text {rel }}=3 V /\left(4 \pi R^{3}\right)$.

The possible separation of the particles, $H$, for any liquid bridge, is limited by lower and upper values. Indeed, if the particles depart beyond a certain distance $H_{\max }$, the bridge becomes unstable due to Rayleigh instabilities [22] and breaks [7, 11, 23], meanwhile if the particles are closer than $H_{m i n}$, the concave geometry is not further preserved.

The minimum distance, $H_{\min }$, is found to be [24]:

$H_{\min }=2 R\left[\frac{2 V_{r e l}+(1-\sin \theta)^{2}(2+\sin \theta)}{3 \cos ^{2} \theta}-(1-\sin \theta)\right]$

For the maximum distance, $H_{\max }$, Lian et al. [25] proposed the following expression for moderate wetting angles:

$H_{\max }=R\left(1+\frac{\theta}{2}\right) \sqrt[3]{\frac{4}{3} \pi V_{\text {rel }}}$

Therefore, a concave liquid bridge is expected to be found only at interparticle distances $H_{\min } \leq H \leq H_{\max }$ for the corresponding values of liquid content and wetting angle.

\section{Calculation of capillary forces}

The capillary force consists of two components. The first is a surface tension term acting at the wetting perimeter, 
tangent to the meniscus at the intersection with the solid surface, and directed towards the liquid. The second component comes from the pressure difference across the curved gas-liquid interface, which can be described by the Laplace-Young equation, computed over the axially projected wetted area of each particle. Thus, the capillary force may be expressed as:

$F_{\text {cap }}=-2 \pi \gamma R \sin \alpha \sin (\alpha+\theta)-\pi \gamma R^{2} \sin ^{2} \alpha\left(\frac{1}{L}-\frac{1}{\rho}\right)$

where the first term corresponds to the wetting force and the second to the Laplace force, being $\gamma$ the surface tension of the liquid. This force can be made dimensionless dividing by the product $(\gamma R)$.

Figure 2 displays some results of the dimensionless capillary force, $F_{c a p} /(\gamma R)$, when $\theta=30^{\circ}$, at different liquid volumes and distances between the solid particles. In general terms, it is observed that the capillary force may show two characters, repulsive and attractive, arising mainly from the behavior of the Laplace component. Indeed, due to the different signs of the principal radii $\rho$ and $L$, this component may be attractive or repulsive. For small distances and small liquid amounts, the Laplacian repulsion is able to dominate over the always attractive wetting force following in repulsive capillary forces. This behavior is quite limited and beyond a certain distance between the solid surfaces, $H_{0}$, the wetting component is higher, and the capillary force becomes attractive. When the liquid volume is larger, the capillary force is attractive in any case, increasing its magnitude for increasing volumes

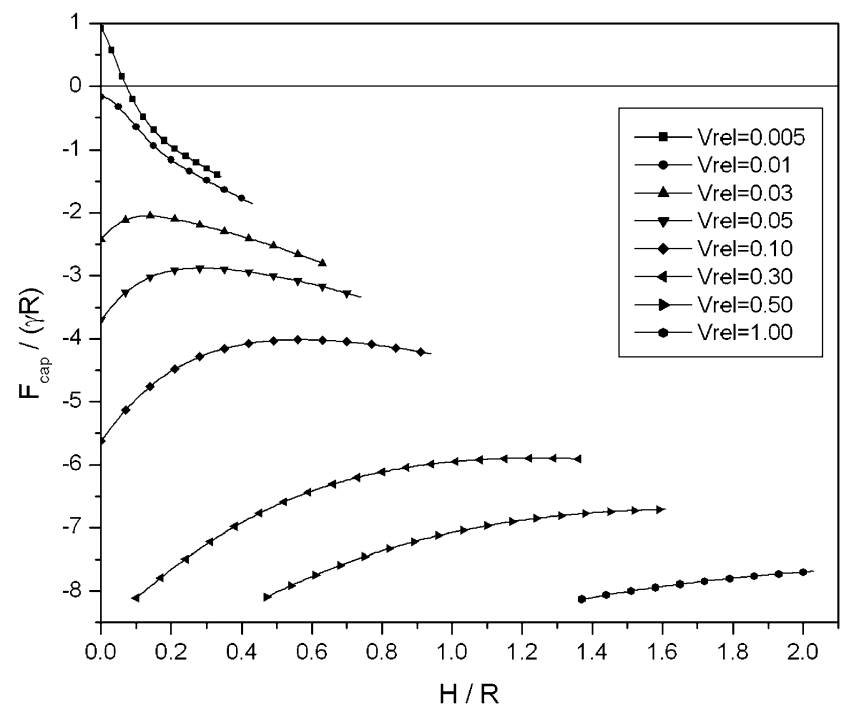

Fig. 2 Scaled capillary force, $F_{c a p} /(\gamma R)$, as a function of the dimensionless interparticle distance, $H / R$, for different liquid contents when $\theta=30^{\circ}$ of liquid due to a higher contact area between the liquid and solid. Repulsive capillary forces have been reported in both theoretical $[25,26]$ and experimental approaches $[3,9]$.

\section{Calculation of van der Waals forces}

When the particles are, like in the present case, only partially covered by the liquid phase, also possessing a dry portion of their surfaces, the typical expression for the van der Waals interaction between particles fully immersed in the liquid, in a proper colloidal suspension, is not longer valid. On contrary, it must be taken into account that the Hamaker constant is different for both wet and dry portions of the particle, thus inducing different potentials between the portions. In addition, the wetted fraction of each solid particle, $d$, (Fig. 1) depends on the separation between the particles, the liquid volume of the bridge, and the wetting angle between the solid and liquid surfaces, which must be calculated as shown above.

In determining the van der Waals interaction, the Derjaguin approximation [27, 28] will be used:

$V_{\text {sphere-sphere }}^{v d W}(H)=\int V_{\text {flat }}(h) d S(h)$

where $V_{f l a t}$ is the interaction potential per unit area between flat surfaces:

$V_{\text {flat }}(h)=-\frac{A}{12 \pi h^{2}}$

and $h$ is the local distance between surface elements, Fig. 1, and $A$ is the Hamaker constant. A method, based on this Derjaguin approximation, the surface element integration, has been proposed $[29,30]$ assuming that this method is not limited by separation distances, particle size, or shape [29]. The distance $h$ can be expressed as:

$h=H+2 R \mp 2 R \sqrt{1-\left(\frac{r}{R}\right)^{2}}$

where the "-"sign corresponds to the two close faces and "+" to the opposite faces. The potential consists out of four terms, arising from the interactions between the two half spheres:

$V^{v d W}(H)=V^{A_{1} A_{1}}+V^{A_{2} A_{2}}-V^{A_{1} A_{2}}-V^{A_{2} A_{1}}$

being:

$V^{A_{1} A_{1}}=-\frac{A}{6} \int_{0}^{R} \sqrt{1-\left(\frac{r}{R}\right)^{2}} \frac{r d r}{\left[H+2 R-2 R \sqrt{1-\left(\frac{r}{R}\right)^{2}}\right]^{2}}$ 
$V^{A_{2} A_{2}}=-\frac{A}{6} \int_{0}^{R} \sqrt{1-\left(\frac{r}{R}\right)^{2}} \frac{r d r}{\left[H+2 R+2 R \sqrt{1-\left(\frac{r}{R}\right)^{2}}\right]^{2}} \quad V^{A_{1} A_{2}}=V^{A_{2} A_{1}}=-\frac{A}{6} \int_{0}^{R} \sqrt{1-\left(\frac{r}{R}\right)^{2}} \frac{r d r}{[H+2 R]^{2}}$

$$
V^{v d W}(H)=-\frac{A}{6} \int_{0}^{R} \sqrt{1-\left(\frac{r}{R}\right)^{2}}\left[\frac{1}{\left[H+2 R-2 R \sqrt{1-\left(\frac{r}{R}\right)^{2}}\right]^{2}}+\frac{1}{\left[H+2 R+2 R \sqrt{1-\left(\frac{r}{R}\right)^{2}}\right]^{2}}-\frac{2}{[H+2 R]^{2}}\right] r d r
$$

Due to the partially wetting of the solid surface, we have to consider that for distances $0 \leq h \leq d$, the surface is wet, and that if $d \leq h \leq R$, the surface is dry. Accordingly, the integral, Eq. 16, has to be cut into two parts:

$V^{v d W}(H)=-\frac{A_{\text {wet }}}{6} \int_{0}^{d} I(r) d r-\frac{A_{d r y}}{6} \int_{d}^{R} I(r) d r$

where $A_{w e t}$ and $A_{d r y}$ are the Hamaker constants for the solid's wet and dry surfaces, respectively, and $I(r)$ is the integrand displayed in Eq. 16. The van der Waals force between a pair of particles is calculated considering the derivative of the potential:

$F_{v d W}=-\frac{\partial V^{v d W}}{\partial H}$

Finally, introducing Eq. 17 into Eq. 18, it is obtained:

$F_{v d W}(H)=\frac{A_{w e t}}{6} \int_{0}^{d} \frac{\partial I(r)}{\partial H} d r+\frac{A_{d r y}}{6} \int_{d}^{R} \frac{\partial I(r)}{\partial H} d r$

The van der Waals force, $F_{v d W}$, has to be determined numerically for the corresponding conditions of $H, V_{\text {rel }}$, and $\theta$. This force can be made dimensionless dividing by the factor $\left(A_{\text {wet }} / R\right)$. Notice that we considered the nonretarded expression of the potential in Eq. 10. In case that the retardation effect is desired to be taken into account, one just needs to write such a version of the potential [30] in Eq. 10 and proceed with the calculations as performed above. Since the effect of the retardation is to decrease the value of the potential with the interparticular distance a bit faster, qualitatively, the following discussion could be extended for such a case.

Figure 3 displays some exemplary cases of the dimensionless van der Waals force, $F_{v d W} /\left(A_{\text {wet }} / R\right)$, as a function of the wetted portion of the particle, $d$, for different interparticle separations, considering that the Hamaker constant for the surface exposed to the air is ten times higher than the constant for the surface in contact with the liquid (water), typical ratio for many materials [31]. As observed, the force is attractive for any value of the wetted portion and decrease in absolute value as $d$ increases, in other words, as the particle is more and more wetted by the liquid, as expected. This means that, in first instance, the van der Waals interaction will be more important in those liquid bridges with the smallest liquid content. The trivial cases are $d=0$ which corresponds to a particle completely dry and $d=R$ corresponding to a particle fully immersed in the liquid. The dependence of $F_{v d W}$ with $d$ is not linear, indicating that it should not be correct to just assume a

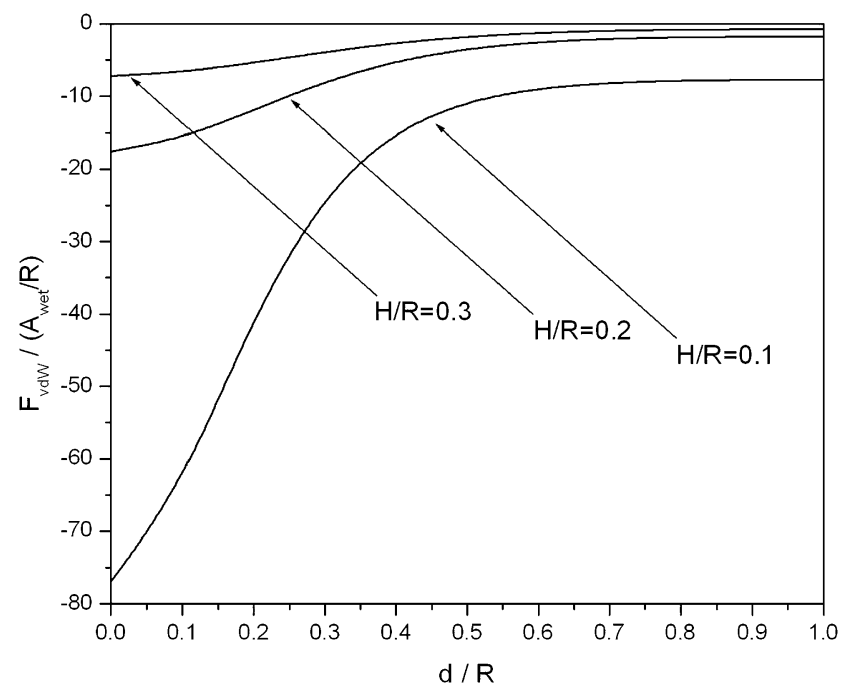

Fig. 3 Scaled van der Waals force, $F_{v d W} /\left(A_{\text {wet }} / R\right)$, as a function of the particle's wetted portion, $d$, divided by the particle's radius, $R$, at different interparticle distances, when $A_{d r y}=10 A_{\text {wet }}$ and $\theta=20^{\circ}$ 


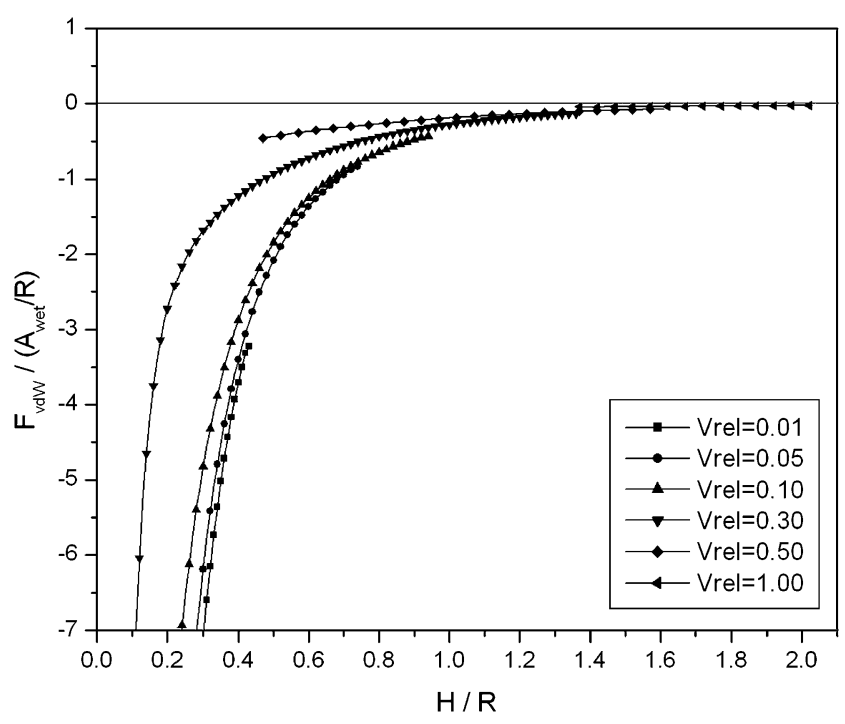

Fig. 4 van der Waals force as a function of the dimensionless interparticles distance, for several relative volume of meniscus liquid, $V_{\text {rel }}$, for a wetting angle $\theta=30^{\circ}$, when $A_{d r y}=10 A_{\text {wet }}$

simple proportion of the van der Waals force from the bulk case.

Recall that the wetted portion of the sphere, $d$, beside the liquid content, $V_{\text {rel }}$, and wetting angle, $\theta$, also depends on the interparticle distance, $H$. When varying the distance between the particles, Fig. 4, it is observed that despite the always attractive character of the van der Waals force, it decreases rapidly as the distance increases, analogous as for the bulk case. Observe the truncation of the plots according to the limiting distances $H_{\min }$, Eq. 6, and $H_{\max }$, Eq. 7, above mentioned. As expected, this force decreases as the amount of liquid increases because the wetted portion of the particles, having a smaller Hamaker constant, also increases.

\section{Comparison between both types of forces}

In order to compare quantitatively both types of forces, let us give some practical values to the parameters involved. Let us consider a particle's size $R=100 \mathrm{~nm}$ and that the liquid of the bridge is water, and the gaseous phase is air, with value of the surface tension, $\gamma=72.8 \mathrm{mN} / \mathrm{m}$. The Hamaker constant for the solid material in contact with water is taken to be $A_{w e t}=10^{-21} \mathrm{~J}$, typical order of magnitude found for e.g., inorganic or ceramic particles $[32,33]$. On view of Figs. 2 and 4, displaying the capillary and van der Waals forces, respectively, it can be stated a priori that the most important forces in this scenario are the capillary ones. Indeed, with the mentioned values, they scale as $\gamma R \approx 10^{-8} \mathrm{~N}$ meanwhile the van der Waals force does it as $A_{w e t} / R \approx 10^{-14} \mathrm{~N}$. Therefore, it is expected a ratio $F_{v d W} / F_{c a p}$ in the order of $10^{-6}$.

Figure 5 shows such a direct comparison for the case $\theta=$ $20^{\circ}$. It is observed that the above prediction is reached at moderate distances between the particles, but for larger ones, the ratio between the forces decreases continuously and strongly, being the van der Waals force seven to eight orders of magnitude smaller than the capillary force. This is the expected behavior since $F_{v d W}$ approaches to zero very fast as the interparticle distance increases.

Nevertheless, there are two situations where both types of forces are comparable in value, or even the van der Waals is higher than the capillary one, determining the force balance: (1) very close approximation of the particles and (2) particles' position such that the capillary force is null, when available.

For checking case 1, Fig. 6 displays some exemplary cases of the total force, $F_{t o t}=F_{c a p}+F_{v d W}$, when $\theta=30^{\circ}$ and several relative liquid volumes, $V_{r e l}$. Observe that the total force shows a deep minimum for $H=0$, arising from the van der Waals' one, meanwhile the capillary force shows a smaller absolute value at this interparticle distance (see inserted magnification in Fig. 6 for the particular case $V_{\text {rel }}=$ 0.005). This means that if $H=0$ is allowed, as soon as the particles approach very close, they fall into this attractive minimum being practically impossible for the particles to leave it. When the interparticle distance, $H$, is a bit larger or the liquid volume is high enough that such close interparticle distances are not possible, the impact of $F_{v d W}$ on $F_{t o t}$ is practically negligible, as indicated by the ratio $F_{v d W}$ $F_{c a p}$ displayed in Fig. 5.

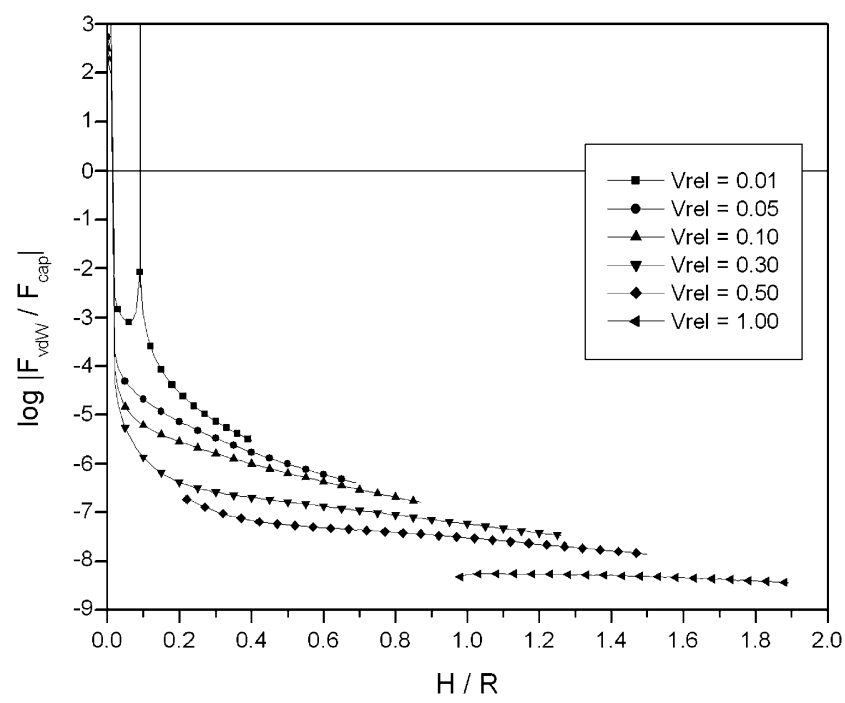

Fig. 5 Ratio $F_{v d W} / F_{c a p}$ as a function of the dimensionless interparticles distance, for several relative volume of meniscus liquid, $V_{r e l}$, being $\theta=20^{\circ}$ and $A_{d r y}=10 A_{\text {wet }}$ 


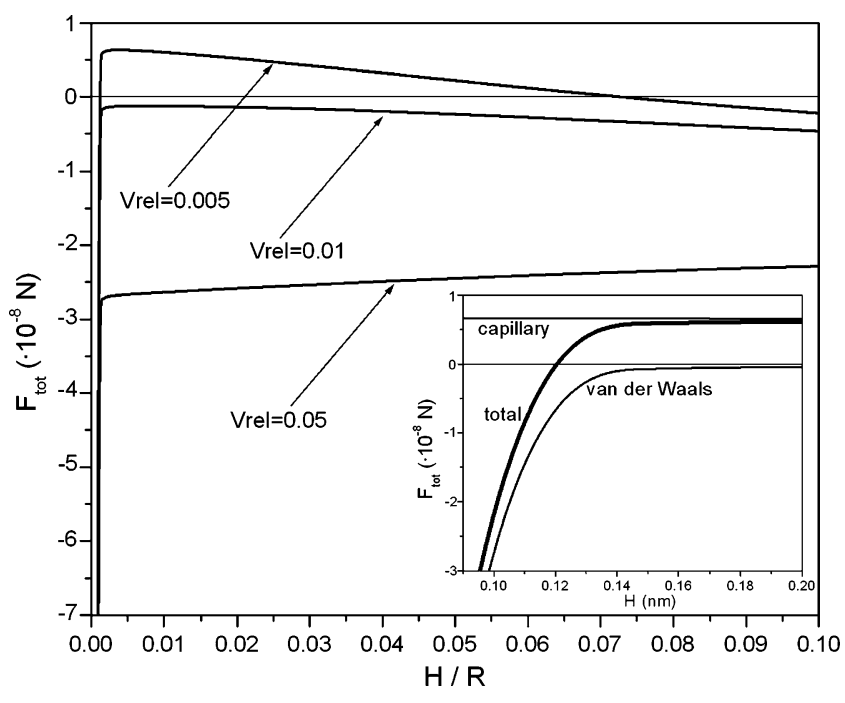

Fig. 6 Total force, as a function of the dimensionless interparticles distance, for several relative liquid volumes, $V_{\text {rel }}$, when $\theta=30^{\circ}$ and $A_{d r y}=10 A_{\text {wet }}$. Inserted, a short distance magnification of the case $V_{r e l}=$ 0.005 , also displaying the van der Waals and capillary forces

Regarding case 2 , when $F_{c a p}=0$, the ratio $F_{v d W} / F_{c a p}$ obviously diverges at the corresponding distance $H_{0}$, Fig. 5 . At this distance, the only acting force is the van der Waals one, whose attractive character makes the particles to attract each other, although weakly. The distances $H_{0}$ are shifted to smaller values, where $F_{\text {tot }}=0$. This is shown in Fig. 7 for $\theta=60^{\circ}$ and $70^{\circ}$. This effect is more pronounced when $\theta$ is very high, where $H_{0}$ are rather small, being less accused, or practically imperceptible, for decreasing angles. Indeed, when $H_{0}$ is longer than $\approx 5 \mathrm{~nm}$, the correction due to the van der Waals interaction is in the second decimal, but on the third to fourth decimal, if such distances are longer than $\approx 10 \mathrm{~nm}$. Since for distances $H<H_{0}$ the capillary force is repulsive, the attractive character of the van der Waals force reduces the range of volumes and distances for which $F_{t o t}>$ 0 . It could be expected a larger gap between both sets of distances for the smaller liquid contents, where the particle' dry portion is larger, and $F_{v d W}$ is higher, but in this case, $F_{c a p}$ is even higher and compensates the effect of $F_{v d W}$. Indeed, the capillary force decreases towards more negative values when $V_{\text {rel }}$ increases, reducing its highness and flattening its plot, Fig. 2.

On the overall, it may be claimed that when determining the forces acting between a pair of particles binded by a liquid bridge, not only the capillary forces have to be calculated, but also the van der Waals force (and the rest of colloidal forces like the electrostatic in charged particles, if considering than they are of the same range of magnitude than those) should be taken into account in case than the particles are separated by very short distances, where $F_{v d W}$ is dominant. For moderate to large interparticle distances, the van der Waals force can be neglected.

\section{Conclusions}

In the present work, we have studied from a numerical approach the scenario displayed when colloidal particles are partially wetted by means of a liquid bridge, considering some of the possible forces that arise in such a situation: capillary and van der Waals force, as representative of the typical colloidal interactions. In order not to introduce more complexity to the system, we have considered the particles uncharged just to avoid electrical interactions and restrict the study to the van der Waals one.

Since both types of forces depend on the geometry of the liquid meniscus, its shape is determined for any value of liquid volume, wetting angle, and interparticle separation. Thus, the principal radii of the meniscus, which mainly govern the capillary force, are calculated, as well as the size of the wetted surface portion of the particles, which splits the contributions from the dry and wet particle areas to the van der Waals force.

The capillary force is found to show both characters, attractive, but also repulsive, the later found to occur in those cases where the liquid content is rather small. As expected, the van der Waals force is always attractive. When comparing in quantitative terms both forces using typical values, it is found that in general terms, the capillary force dominates over the van der Waals. The later pulls down the total force, sum of both, to more negative values, but this effect is practically negligible for moderate or long distances between the particles. Nevertheless, the contribution of the van der Waals force is noticeable and even dominant at very short distances. Indeed, it induces a deep minimum at distances close to zero, not present for the

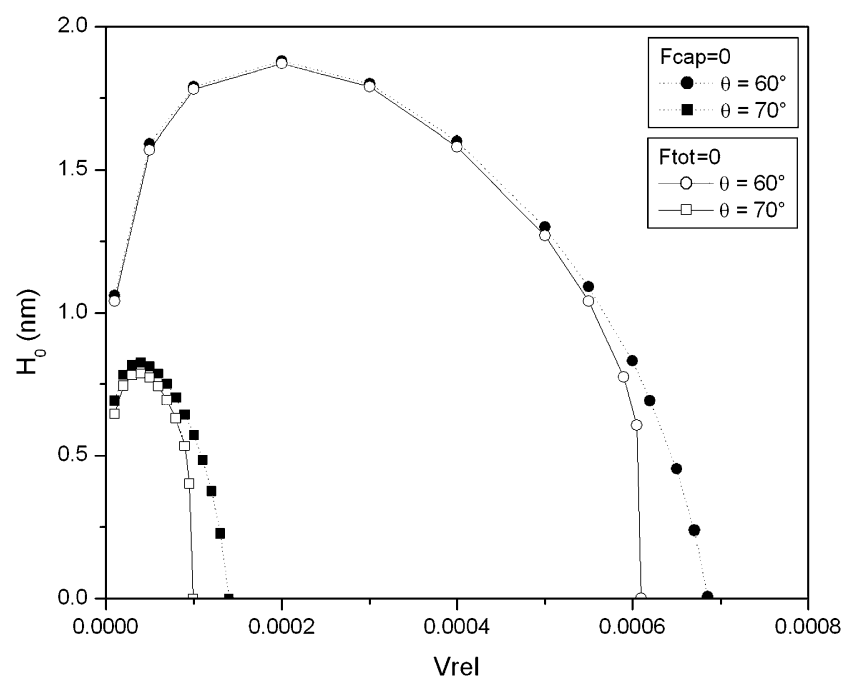

Fig. 7 Interparticles distances, $H_{0}$, at which $F_{c a p}$ and $F_{t o t}$ are zero, as a function of the relative volume of meniscus liquid, $V_{\text {rel }}$, for wetting angles $\theta=60^{\circ}$ and $70^{\circ}$ 
capillary force alone. Also, $F_{v d W}$ shifts to smaller ones the distances, $H_{0}$, at which $F_{c a p}=0$, this effect more evident for the highest wetting angles at the shorter distances.

\section{References}

1. Haines WB (1925) Studies in the physical properties of soils. J Agric Sci 15:529-543

2. Fischer RA (1926) On the capillary forces in an ideal soil: correction of the formulae given by W.B. Haines. J Agric Sci 16:492-505

3. Huppmann WJ, Rieger H (1975) Modelling of rearrangement processes in liquid phase sintering. Acta Met 23:965-971

4. Pietsch W, Rumpf H (1967) Haftkraft, Kapillardrudr, Fliissigkeitsvolumen und Grenzwinkel einer Fliissigkeitsbriicke zwischen zwei Kugeln. Chemie Ing Techn 39:885-893

5. Kralchevsky PA, Nagayama K (2001) Particles at fluid interfaces. Elsevier, Amsterdam

6. Heady RB, Cahn JW (1970) An analysis of capillary forces in liquid-phase sintering of spherical particles. Metall Trans 1:185189

7. Erle MA, Dyson DC, Morrow NR (1971) Liquid bridges between cylinders, in a torus, and between spheres. AIChE J 17:115-121

8. Hotta K, Takeda K, Iinoya K (1974) Capillary binding force of a liquid bridge. Powder Technol 10:231-242

9. Orr FM, Scriven LE, Rivas AP (1975) Pendular rings between solids - meniscus properties and capillary force. J Fluid Mech 67:723-742

10. Mehrotra VP, Sastry KVS (1980) Pendular bond strength between unequal-sized spherical-particles. Powder Technol 25:203-214

11. De Bisschop FRE, Rigole WJL (1982) A physical model for liquid capillary bridges between adsorptive solid spheres - the nodoid of Plateau. J Colloid Interf Sci 88:117-128

12. Bayramli E, Abou-Obeid A, van de Ven TGM (1987) Liquid bridges between spheres in a gravitational field. J Colloid Interf Sci 116:490-502

13. Eremenko VN, Naidich YV, Lavrinenko IA (1970) Liquid-phase sintering. Consultants Bureau, New York

14. Groger T, Tuzun U, Heyes DM (2003) Modelling and measuring of cohesion in wet granular materials. Powder Technol 133:203215

15. Gonzenbach UT, Studart AR, Tervoort E, Gauckler LJ (2006) Stabilization of foams with inorganic colloidal particles. Langmuir 22:10983-10988
16. Willet CD, Adams MJ, Johnson SA, Seville JPK (2003) Effects of wetting hysteresis on pendular liquid bridges between rigid spheres. Powder Technol 130:63-69

17. Meurisse MH, Querry M (2006) Squeeze effects in a flat liquid bridge between parallel solid surfaces. J Tribol 128:575-584

18. Mazzone DN, Tardos GI, Pfeffer R (1986) The effect of gravity on the shape and strength of a liquid bridge between 2 spheres. J Colloid Interf Sci 113:544-556

19. Dai ZF, Lu SC (1998) Liquid bridge rupture distance criterion between spheres. Int J Mineral Proc 53:171-181

20. Delaunay C (1841) Sur la surface de revolution dont la courbure moyenne est constante. J Math Pures Appl 6:309-314

21. Delannay F (2005) Modelling of the influence of dihedral angle, volume fractions, particle size and coordination on the driving forces for sintering of dual phase systems. Phil Mag 85:3719-3733

22. Rayleigh FRS (1892) On the instability of a cylinder of viscous liquid under capillary force. Phil Mag 207:145-154

23. Denkov ND, Ivanov IB, Kralchevsky PA, Wasan DT (1992) A possible mechanism of stabilization of emulsions by solid particles. J Colloid Interf Sci 150:589-593

24. Megias-Alguacil D, Gauckler LJ (2009) Capillary forces between two solid spheres linked by a concave liquid bridge: regions of existence and forces mapping. AIChE J 55:1103-1109

25. Lian GP, Thornton C, Adams MJ (1993) A theoretical study of the liquid bridge forces between two rigid spherical bodies. J Colloid Interf Sci 161:138-147

26. Anestiev LA, Froyen LJ (1999) Model of the primary rearrangement processes at liquid phase sintering and selective laser sintering due to biparticle interactions. Appl Phys 86:4008-4017

27. Derjaguin BV (1934) Analysis of friction and adhesion IV. The theory of the adhesion of small particles. Kolloid Z 69:155-164

28. Martinez-Lopez F, Cabrerizo-Vilchez MA, Hidalgo-Alvarez R (2000) Colloidal interaction at the air-liquid interface. J Colloid Interf Sci 232:303-310

29. Bhattacharjee S, Elimenech M (1997) Surface Element Integration: a novel technique for evaluation of DLVO interaction between a particle and a flat plate. J Colloid Interf Sci 193:273-285

30. Bhattacharjee S, Elimenech M, Borkovec M (1998) DLVO interaction between colloidal particles: beyond Derjaguin's approximation. Croat Chem Acta 4:883-903

31. Lyklema K (2000) Fundamentals of interface and colloidal science. Academic, London

32. Megias-Alguacil D, Duran JDG, Delgado AV (2000) Yield stress of concentrated zirconia suspensions: correlation with particle interactions. J Colloid Interf Sci 231:74-83

33. French RH (2000) Origins and applications of London dispersion forces and Hamaker constants in ceramics. J Am Ceram Soc $83: 2117-2146$ 